\title{
Efficacy and safety profile of S-1 in patients with metastatic gastric cancer in clinical practice: results from a post-marketing survey
}

\author{
Hiroki Kawai, Atsushi Ohtsu, Narikazu Boku, Yasuo Hamamoto, Fumio Nagashima, Manabu Muto, \\ Yasushi Sano, Kiyomi Mera, Tomonori Yano, Toshiniko Doi, and Shigeaki Yoshida \\ Division of Digestive Endoscopy and Gastrointestinal Oncology, National Cancer Center Hospital East, 6-5-1 Kashiwanoha, Kashiwa, \\ Chiba 277-8577, Japan
}

\begin{abstract}
Background. S-1(TS-1 $\left.{ }^{\circledR}\right)$, a novel oral fluoropyrimidine, has been commercially available for gastric cancer in Japan. A nationwide post-marketing survey for safety was carried out after its approval. The aim of this analysis was to evaluate the efficacy and safety profile of this agent in clinical practice for patients with advanced gastric cancer registered in the postmarketing survey from our institution.

Methods. Between April 1999 and April 2000, a total of 51 chemo-naive patients were registered in the survey from the National Cancer Center Hospital East. S-1 was administered at $80 \mathrm{mg} / \mathrm{m}^{2} /$ day for 4 weeks, followed by a 2-week rest, repeated every 6 weeks until disease progression, unacceptable toxicity, or the patient's refusal.

Results. Of the 51 patients, $41(80 \%)$ fulfilled the criteria of the guidelines determined by the company as appropriate patients for the drug administration. The median number of treatment courses was five. Toxicities were generally mild: grade 3 or 4 toxicities were seen in $10 \%$ or fewer patients, and no treatment-related deaths occurred. In the 47 patients with evaluable lesions, there were 2 complete responses and 18 partial responses, with a response rate of $43 \%$. With a minimum follow-up of 2 years, median survival time and 2-year survival were 11.1 months and $33 \%$, respectively. The majority of the 17 2-year survivors had diffuse-type histology and peritoneal metastasis and achieved an objective response.

Conclusion. S-1 appears to be safe and highly active, with favorable longterm survival in patients with metastatic gastric cancer, particularly in those with diffuse-type histology and peritoneal metastasis.
\end{abstract}

Key words Gastric cancer · Chemotherapy · S-1

\section{Introduction}

Despite the remarkable improvements in survival gained by early detection and curative surgery in pa-

Offprint requests to: A. Ohtsu

Received: September 26, 2002 / Accepted: January 9, 2003 tients with locoregional gastric cancer in Japan, the survival of patients with unresectable or recurrent disease still remains poor. Various treatments, including combination chemotherapy, have been attempted for this disease. Although systemic chemotherapy has a survival benefit as compared with best supportive care [1-3], this advantage is marginal, and the development of new active agents is indispensable to overcome this circumstance.

$\mathrm{S}-1$ is a novel oral fluoropyrimidine, based on the biochemical modulation of 5-flnorouracil (FU); it contains tegafur (FT), gimestat (CDHP), and otastat potassium (Oxo) at a molar ratio of FT:CDHP:Oxo = 1:0.4:1, and was developed by Taiho Pharmaceutical Company, Tokyo [4]. In the two registration phase II studies in Japan, this agent demonstrated excellent activity for gastric cancer, with response rates of $49 \%$ $(25 / 51)$ and $40 \%(20 / 50)$, respectively $[5,6]$. The toxicity profile of this agent was mild, with a less than $10 \%$ incidence of grade 3 or 4 . Based on these results, this agent was approved by the Ministry of Health, Labor, and Welfare in Japan in 1999. However, the Ministry recommended that the pharmaceutical company undertake a nationwide post-marketing survey of up to 3000 patients treated with this agent to monitor the patients and to find whether there were any serious toxicities, as early as possible. During the period between April 1999 and April 2000, more than 3000 patients nationwide were registered with the pharmaceutical company, with the requirement that case report forms be submitted. This survey revealed the safety of this agent, and this was followed by a marked increase in its use in clinical practice in Japan. However, precise information, particularly on its efficacy, has not been clarified yet.

The aim of this analysis was to evaluate the efficacy and toxicity of S-1 in clinical practice, based on the investigation of treatment outcomes in patients from our institution registered in the survey. 


\section{Patients and methods}

\section{Source of the patients}

During the period of the post-marketing survey described in the "Introduction", the pharmaceutical company restricted the supply of this agent to those patients in whom it was appropriate according to the following criteria: Eastern Cooperative Oncology Group performance status of two or better; unresectable or recurrent gastric cancer; WBC, 3500-12000/ $\mu 1$; neutrocytes, 2000 or more $\mu \mathrm{l}$; hemoglobin, 9.0 or more $\mathrm{g} / \mathrm{dl}$; platelets, 100000 or more $\mu \mathrm{l}$; total bilirubin, as partate aminotransferase (AST), and alanine aminotransferase (ALT), within two times the upper limit, serum creatinin, within the normal upper limit. However, if the oncologist at each institution considered that the patient could safely be administered this agent, despite not fulfilling any one of the above criteria, this agent was provided by the company.

Between April 1999 and April 2000, more than 3000 patients nation-wide were registered with the company and were treated with this agent. A total of 91 patients with advanced gastric cancer were registered in the survey from the National Cancer Center Hospital East. Of the 91 patients, 51 were chemo-naive and were the subjects of this study.

\section{Treatment schedule}

S-1 was administered according to the treatment schedule in the registration study $[5,6]$. The initial doses were assigned on the basis of body surface area (BSA) as follows: BSA less than $1.25 \mathrm{~m}^{2}, 80 \mathrm{mg} /$ day; BSA, $1.25 \mathrm{~m}^{2}$ to $1.5 \mathrm{~m}^{2}, 100 \mathrm{mg} /$ day; BSA more than $1.5 \mathrm{~m}^{2}, 120 \mathrm{mg} /$ day. The treatment schedule consisted of twice daily administrations, after breakfast and dinner, for 28 consecutive days, followed by a 2-week rest. This schedule was repeated every 6 weeks until the occurrence of disease progression, unacceptable toxicity, or the patient's refusal. Dose modification and treatment interruption were carried out based on the protocol in the registration trials $[5,6]$.

\section{Evaluation}

Baseline evaluation included complete medical history, physical examination, complete blood cell count, serum chemistry, gastroscopy, abdominal computed tomography (CT) scan, and chest X-ray. Blood chemistry, and subjective/objective symptoms of toxicity were monitored on a 2-weekly basis during the treatment. Toxicity was graded according to the National Cancer Institute Common Toxicity Criteria during all of the treatment courses. To assess an objective response, the lesions were basically evaluated every 2 months. For primary gastric tumors, the response was evaluated according to the criteria proposed by the Japanese Research Society for Gastric Cancer [7], using gastrography or gastroscopy. The responses of metastatic lesions were assessed according to World Health Organization (WHO) criteria [8], using CT scans.

\section{Statistics}

Survival time was calculated from the initial date of the first course of chemotherapy to the date of death from any cause, or to the date of the last confirmation of survival, using the Kaplan-Meier method. To compare survivals between subgroups, the log-rank test was used to evaluate statistical significance.

\section{Results}

\section{Patient characteristics}

Of the 51 patients registered, 41 (80\%) patients fulfilled the criteria of appropriate patients determined by the company, and the remaining $10(20 \%)$ patients did not fulfill the criteria, due to the following factors: lower WBC or neutrocyte count in 2, lower hemoglobin in 2, lower platelet count in 1 , slight impairment of liver function in 3, and slight impairment of renal function in 2 patients. The baseline characteristics of the 51 patients are listed in Table 1 . The median age was 59 years (range, 28-78 years). Most of the patients had a good performance status, of 0 or 1 . Twenty-seven $(53 \%)$ patients had had a prior history of gastrectomy before the chemotherapy was initiated. Macroscopically, 10 (20\%) patients had scirrhous type of primary tumor. On histology, 18 (35\%) patients had intestinal-type and 32 (63\%) patients had diffuse-type adenocarcinoma. There were $31(61 \%)$ patients having only a single metastatic site and the remaining $20(39 \%)$ patients had two or more metastatic sites. Major metastatic sites were abdominal nodes in 26 patients, peritoneum in 24 , and the liver in 17. Of the 24 patients with peritoneal metastases, 13 patients were diagnosed at laparotomy or laparoscopy and the remaining 11 patients at preoperative imaging, such as barium enema or CT scan. Thirty-three $(65 \%)$ patients could normally take solid foods and the other $18(35 \%)$ patients had impairment of oral intake, including 1 patient who had required nutritional support with hyperalimentation at registration. All patients were treated as outpatients during most of the treatment period. The median number of treatment courses administered was 5, ranging from 1 to 19 . After failure of this treatment, second-line chemotherapy was administered to $37(73 \%)$ patients. 
Table 1. Patient characteristics

Age (years); median (range)

Sex Male

Female

$59(28-78)$

ECOG performance status

0

1

2

History of gastrectomy $(+/-)$

Macroscopic type of primary site Scirrhous

Nonscirrhous

Unknown

Histological type

Intestinal type

Diffuse type

Unknown

Number of metastatic sites

1

2

3

Metastatic site

Abdominal lymph node 26

Peritoneum 24

Liver

Others

ECOG, Eastern Cooperative Oncology Group

Table 2. Toxicity

\begin{tabular}{|c|c|c|c|c|c|}
\hline & \multicolumn{4}{|c|}{ Grade } & \multirow{2}{*}{$\begin{array}{c}\geqq \text { Grade } 3 \\
(\%)\end{array}$} \\
\hline & 1 & 2 & 3 & 4 & \\
\hline Leukopenia & 14 & 11 & 1 & 0 & 2 \\
\hline Neutropenia & 13 & 7 & 2 & 0 & 4 \\
\hline Anemia & 16 & 23 & 3 & 2 & 10 \\
\hline Thrombocytopenia & 4 & 2 & 2 & 0 & 4 \\
\hline AST & 15 & 1 & 2 & 0 & 4 \\
\hline ALT & 11 & 3 & 0 & 0 & 0 \\
\hline Bilirubin & 15 & 10 & 2 & 0 & 4 \\
\hline Creatinine & 4 & 1 & 1 & 0 & 2 \\
\hline Nausea & 9 & 5 & 0 & 0 & 0 \\
\hline Anorexia & 12 & 7 & 1 & 0 & 2 \\
\hline Diarrhea & 8 & 2 & 0 & 0 & 0 \\
\hline Skin rash & 9 & 1 & 0 & 0 & 0 \\
\hline Pigmentation & 7 & 0 & 0 & 0 & 0 \\
\hline
\end{tabular}

\section{Toxicity}

Table 2 summarizes the toxicity observed during all the treatment courses. Toxicities were generally mild: grade 3 or 4 toxicities were observed in $10 \%$ or fewer patients. Grade 4 toxicites were seen in only 2 (4\%) patients with anemia, which seemed to be partly caused by the primary disease. Of the 51 patients, only $1(2 \%)$ required hospitalization due to toxicity; this patient had grade 3 anorexia during the sixth course, but was discharged 3

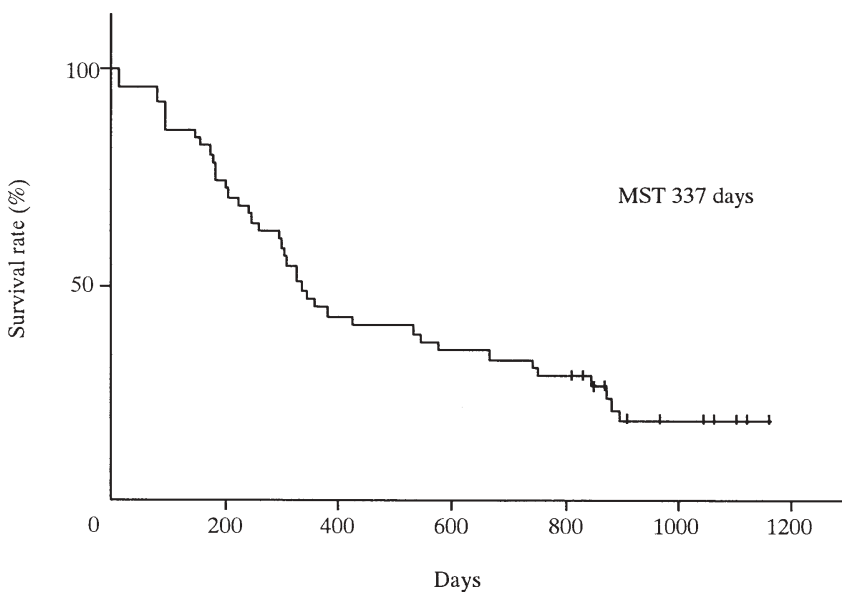

Fig. 1. Overall survival of the 51 chemo-naive patients treated with S-1. MST, Median survival time

days after the hospitalization with recovery from the toxicity. There were $3(6 \%)$ patients who died within 30 days after completion of the treatment due to tumor progression, and no treatment-related deaths occurred.

\section{Response and survival}

Of the 51 patients, $2(4 \%)$ patients achieved complete responses (CRs) and 18 (35\%) patients achieved partial responses (PRs), with a response rate of $39 \%$ (20/51) on an intent-to-treat basis; the response rate was $43 \%$ (20/ 47 ) in patients with evaluable lesions. The response rate of the primary sites as evaluated by the Japanese criteria was $42 \%(10 / 24)$, including $1 \mathrm{CR}$. With a median follow-up period in survivors of 2.6 years and a minimum of 2 years, the median survival time (MST) of all patients was 11.1 months (Fig. 1). One-, 2-, and 3-year survival rates were $45 \%, 33 \%$, and $19 \%$, respectively.

There were 17 patients who survived for longer than 2-years, and their characteristics are listed in Table 3. All of the 17 patients had a good PS, of 0 or 1 . Of these 17 patients, 13 (76\%) had diffuse-type adenocarcinoma on histology: 13 (41\%) of the 32 patients with diffuse type at baseline have survived for longer than 2 years, while $4(22 \%)$ of the 18 patients with intestinal type were 2-year survivors, although there were no significant differences in survival between patients with intestinal and with diffuse type $(P=0.40)$. In terms of metastatic sites, in the 17 patients who survived for longer than 2 years, there were 11 patients with peritoneal, 7 with abdominal lymph nodes, and 4 with liver metastases: 11 (46\%) of the 24 patients with peritoneal metastasis at baseline, 7 (27\%) of the 26 with abdominal lymph nodes, and 4 (24\%) of the 17 with liver metastasis have survived for longer than 2 years. In the 14 of the 17 patients who survived for longer than 2 years and who 
Table 3. Two-year survivors treated with S-1

\begin{tabular}{|c|c|c|c|c|c|c|c|c|c|}
\hline $\begin{array}{l}\text { Age } \\
\text { (years) }\end{array}$ & Sex & PS & $\begin{array}{l}\text { Macroscopic type } \\
\text { of primary site }\end{array}$ & Histology & Gastrectomy & $\begin{array}{c}\text { Site of } \\
\text { metastasis }\end{array}$ & $\begin{array}{c}\text { Response } \\
1^{\text {st }} / 2^{\text {nd }}\end{array}$ & $\begin{array}{l}\text { Survival } \\
\text { (Days) }\end{array}$ & $\begin{array}{c}\text { Present } \\
\text { status }\end{array}$ \\
\hline 56 & $\mathrm{~F}$ & 0 & Scirrhous & Diffuse & + & Peritoneum & $\mathrm{NE}-$ & 1162 & Alive \\
\hline 44 & $\mathrm{~F}$ & 0 & Scirrhous & Diffuse & + & Peritoneum & $\mathrm{NE} \mathrm{NC}$ & 1128 & Alive \\
\hline 33 & $\mathrm{M}$ & 0 & Non-scirrhous & Diffuse & - & Peritoneum & PR NC & 1110 & Alive \\
\hline 28 & $\mathrm{~F}$ & 0 & Scirrhous & Diffuse & - & Peritoneum, A-LN & PR NC & 1066 & Alive \\
\hline 65 & M & 0 & Non-scirrhous & Intestinal & + & Peritoneum & $\mathrm{NE}-$ & 1048 & Alive \\
\hline 61 & M & 1 & Non-scirrhous & Diffuse & - & Peritoneum, A-LN & PR NC & 979 & Alive \\
\hline 63 & $\mathrm{M}$ & 1 & Non-scirrhous & Diffuse & + & A-LN & PR NC & 924 & Alive \\
\hline 68 & M & 0 & Non-scirrhous & Diffuse & + & Liver & PR NC & 896 & Dead \\
\hline 54 & $\mathrm{~F}$ & 1 & Non-scirrhous & Diffuse & - & Liver & CR NC & 884 & Dead \\
\hline 59 & $\mathrm{~F}$ & 1 & Non-scirrhous & Diffuse & - & Peritoneum & NC PD & 874 & Dead \\
\hline 78 & $\mathrm{M}$ & 0 & Non-scirrhous & Intestinal & - & A-LN & PR PR & 869 & Alive \\
\hline 67 & M & 0 & Non-scirrhous & Intestinal & - & Peritoneum, Liver & PR PD & 857 & Alive \\
\hline 66 & $\mathrm{~F}$ & 0 & Non-scirrhous & Diffuse & + & A-LN & $\mathrm{CR}-$ & 848 & Dead \\
\hline 54 & $\mathrm{~F}$ & 0 & Scirrhous & Diffuse & - & Peritoneum & $\mathrm{NC} \mathrm{NC}$ & 837 & Alive \\
\hline 53 & M & 0 & Non-scirrhous & Diffuse & - & Peritoneum, A-LN & $\mathrm{NC} \mathrm{NC}$ & 804 & Alive \\
\hline 58 & M & 0 & Non-scirrhous & Intestinal & + & Liver, A-LN & PR NC & 752 & Dead \\
\hline 45 & $\mathrm{~F}$ & 0 & Non-scirrhous & Diffuse & + & Peritoneum & PR PR & 745 & Dead \\
\hline
\end{tabular}

A-LN, Abdominal lymph node; PS, performance status; NE, not evaluated; NC, no change; PR, partial response; CR, complete response; PD, progressive disease

had evaluable lesions, 11 (79\%) patients achieved objective responses, including $2 \mathrm{CRs}$, with this treatment.

\section{Discussion}

In the approval system for anticancer agents in Japan, new agents are usually approved by the results of two independent registration phase II studies, while a postmarketing survey for evaluating safety and clinical trials including randomized phase III trials for evaluating efficacy are recommended after the approval. S-1 is a newly developed fluoropyrimidine and has been commercially available in Japan since March 1999, based on high response rates with low toxicity in the two registration phase II studies for gastric cancer [5,6]. According to the above guidance, a nationwide post-marketing survey was carried out in up to 3000 patients and two randomized controlled trials evaluating the efficacy of this agent are now underway in Japan. In addition, a small combination study with another agent provided promising results for gastric cancer [9], and S-1 also showed activity for other malignancies, such as head and neck [10], colorectal [11], breast [12], and lung cancers [13] in single agent studies.

There are some non-negligible issues in the field of gastric cancer chemotherapy in this approval system. Usually, a phase II study requires measurable metastatic lesions to determine a response rate as a primary endpoint. However, patients with peritoneal metastasis, which is the major metastatic site of advanced or recurrent gastric cancer, usually do not have measurable metastatic lesions and are excluded from a phase
II study. These circumstances yield a paradoxical phenomenon, that a new agent for gastric cancer is approved without an evaluation of efficacy for the majority of patients with this disease. In addition, peritoneal metastasis often causes bowel obstruction and hydronephrosis, which may prolong the elimination of an anticancer agent and induce serious toxicity. Therefore, newly available agents for gastric cancer should be carefully re-evaluated for efficacy and safety when indicated for into clinical practice.

The nationwide post-marketing survey of S-1 has confirmed that this agent is safe, particularly when used as indicated for appropriate patients, as defined in "Patients and methods" [14]. In the present analysis, although $20 \%$ of the patients had characteristics incompatible with one of the criteria for appropriate patients at baseline, the toxicity profile revealed the safety of this agent in clinical practice, as shown in the previous registration trials. However, this agent contains CDHP, which reduces 5-FU degradation by the inhibition of dihydropyrimidine dehydrogenase, and this compound is eliminated from the kidney. Multivariate analysis in the post-marketing survey of more than 3000 patients revealed that grade 3 or worse hematological toxicities occurred significantly more frequently in patients with impairment of renal function. Also, in the present analysis, one of the two patients with a slight increase of serum creatinine developed grade 3 thrombocytopenia requiring platelet infusion. Thus, this agent should be carefully administered, with dose reductions if required, for patients with impairment of renal function.

In respect to efficacy, the present results seemed to be promising, with a high response rate (the same as that 
seen in the registration trials) and favorable survival rates, particularly a median survival of 11.1 months and actual 2-year survival of $33 \%$, compared with the findings in various other reports, including those of combination regimens, in which findings usually ranged from 6 to 10 months for median and around $10 \%$ for 2-year survival $[15,16]$. However, there could be some selection biases in the present population. One of the major biases arises because this agent is in an oral form: patients who had poor oral intake were automatically excluded as subjects to receive this agent. Further, more than half of the patients in the present population had a prior gastrectomy and histologically diffuse type. In addition, the present results were only the experience in a single institution. These biases might have produced the favorable survival in the present study. However, the patients in this study were prospectively registered with the company, and in particular, the actual 2-year survival rate of $33 \%$ seems to be markedly higher than we have ever experienced at our institution in clinical practice using other regimens $[17,18]$. In particular, most of the 2-year survivors in the present analysis had diffuse-type histology with peritoneal metastasis. These results suggested that this agent would provide favorable outcomes for such major populations in clinical practice and may be suitable in an adjuvant setting, although this should be evaluated in an ongoing randomized study in Japan.

This agent appears to have various advantages for gastric cancer patients: convenient oral form, high response rate even for the primary site, mild toxicity, good compliance to continue this treatment, and a high frequency of patients able to receive second-line chemotherapy. These advantages might yield such a favorable longterm survival. Of course these should be evaluated in a prospective randomized controlled trial. In Japan, three randomized trials that include this agent are now underway in patients with metastatic disease: 5-FU alone versus cisplatinum + irinotecan (CPT-11) versus S-1 alone (the Japan Clinical Oncology Group study), and S-1 alone versus S-1 + cisplatinum, and 5-FU+ leucovorin versus S-1 alone as industrial companysponsored studies. These studies will clarify the true impact of this agent in patients with metastatic gastric cancer. Further evaluations are still needed.

\section{References}

1. Murad AM, Santiago FF, Petroianu A, et al. Modified therapy with 5-fluorouracil, doxorubicin, and methotrexate in advanced gastric cancer. Cancer 1993;72:37-41.
2. Glimelius B, Hoffman K, Haglund U, et al. Initial or delayed chemotherapy with best supportive care in advanced gastric cancer. Ann Oncol 1994;5:189-90.

3. Pyrhonen S, Kuitunen T, Nyandoto P, et al. Randomized comparison of fluorouracil epidoxorubicin and methotrexate (FEMTX) plus supportive care with supportive care alone in patients with non-resectable gastric cancer. Br J Cancer 1995;71: 587-91.

4. Shirasaka T, Shimamoto Y, Ohshimo H, et al. Development of a novel form of an oral fluorouracil derivative (S-1) directed to the potentiation of the tumor selective cytotoxicity of 5-fluorouracil by two biochemical modulations. Anticancer Drugs 1996;7:54857.

5. Sakata Y, Ohtsu A, Horikoshi N, et al. Late phase II study of novel oral fluoropyrimidine anticancer drug S-1 (1M tegafur $-0.4 \mathrm{M}$ gimestat- $1 \mathrm{M}$ otastat potassium) in advanced gastric cancer patients. Eur J Cancer 1998;34:1715-20.

6. Koizumi W, Kurihara M, Nakano S, et al. Phase II study of S-1, a novel oral derivative of 5-fluorouracil, in advanced gastric cancer. Oncology 2000;58:191-7.

7. Japanese Research Society for Gastric Cancer. Japanese classification of gastric carcinoma. 1st English Ed. In: Japanese Research Society for Gastric Cancer, editors. Tokyo: Kanehara; 1995.

8. World Health Organization. WHO Handbook for reporting results of cancer treatment. WHO Offset Publication No. 48. Geneva: World Health Organization; 1979.

9. Ohtsu A, Boku N, Nagishima F, et al. A phase I/II study of S-1 plus cisplatin (CDDP) in patients (pts) with advanced gastric cancer (AGC) (abstract). Proc Am Soc Clin Oncol 2001;20:165.

10. Inuyama $\mathrm{Y}$, Kida A, Tsukuda M, et al. Late phase II study of S-1 in patients with advanced head and neck cancer (in Japanese). Gan to Kagakuryouhou (Jpn J Cancer Chemother) 2001;28: 1381-90.

11. Ohtsu A, Baba H, Sakata Y, et al. Phase II study of S-1, a novel oral fluoropyrimidine derivative, in patients with metastatic colorectal carcinoma. Br J Cancer 2000;83:141-5.

12. Sano M, Saeki T, Takashima S, et al. Late phase II study of S-1 in patients with advanced and/or recurrent breast cancer (abstract). Proc Am Soc Clin Oncol 2000;19:105.

13. Kawahara M, Furuse K, Segawa Y, et al. Phase II study of S-1, a novel oral fluorouracil in advanced non-small-cell lung cancer. $\mathrm{Br}$ J Cancer 2001;85:939-43.

14. Nagashima F, Ohtsu A, Boku N, et al. Japanese nation-wide post marketing survey of S-1 in patients with advanced gastric cancer. Proc Am Soc Clin Oncol 2002;21:(abstract no. 2228).

15. Ohkuwa M, Ohtsu A, Boku N, et al. Long-term results for patients with unresectable gastric cancer who received chemotherapy in the Japan Clinical Oncology Group (JCOG) trials. Gastric Cancer 2000;3:145-50.

16. Ohtsu A, Shimada Y, Shirao K, et al. Randomized phase III trial of 5-fluorouracil alone versus 5-fluorouracil plus cisplatin versus uracil and tegafur plus mitomycin $\mathrm{C}$ in patients with unresectable advanced gastric cancer: the Japan Clinical Oncology Group Study (JCOG9205). J Clin Oncol 2003;21:54-9.

17. Yoshida M, Boku N, Ohtsu A, et al. Combination chemotherapy of irinotecan plus cisplatin for advanced gastric cancer: efficacy and feasibility in clinical practice. Gastric Cancer 2001;4:144-9.

18. Tahara M, Ohtsu A, Boku N, et al. Sequential methotrexate and 5 -fluorouracil therapy for gastric cancer patients with peritoneal dissemination: a retrospective study. Gastric Cancer 2001;4:212-8. 